\title{
Profound Hypothermia Secondary to Clobazam Use in Epilepsy: A Novel Association
}

\author{
Marc P. DiFazio, Bennet Lavenstein, Scott Demarest \\ Neurology, Children's National Medical Center, Washington DC, USA \\ Email: mdifazio@childrensnational.org, blavenst@childrensnational.org, sdemares@childrensnational.org
}

Received 3 December 2013; revised 2 January 2014; accepted 1 February 2014

Copyright (C) 2014 by authors and Scientific Research Publishing Inc.

This work is licensed under the Creative Commons Attribution International License (CC BY).

http://creativecommons.org/licenses/by/4.0/

(c) (i) Open Access

\begin{abstract}
Clobazam, a 1-5 benzodiazepine, was introduced in the 1970s for the treatment of anxiety and agitation. Antiepileptic properties were recognized, and efficacy in a number of epilepsy syndromes was demonstrated in humans, with good tolerance. Recent reviews are generally favorable, with a relative minimum of medication-related side effects. However, a number of benzodiazepines have been associated with causing hypothermia. To date, this side effect has not been reported with clobazam. We report two cases of profound hypothermia associated with the use of this medication for the treatment of epilepsy. Both children had significant cerebral dysgenesis and were developmentally impaired, but neither had experienced hypothermia before. Temperature dysregulation was resolved with medication withdrawal after an extensive work-up for alternative causes. Hypothermia should be considered as a possible side effect of clobazam, although the exact mechanism is unknown. Appropriate monitoring of temperature is appropriate, and precautions should be offered by caregivers.
\end{abstract}

\section{Keywords}

Clobazam; Epilepsy; Hypothermia

\section{Introduction}

Clobazam, a 1-5 benzodiazepine, was introduced in the 1970s for the treatment of anxiety and agitation [1] [2]. It was distinguished from benzodiazepines available at the time by demonstrated efficacy in anxiolysis, but with minimal if any effects on performance function [3]. Antiepileptic properties were recognized in animal studies, and efficacy in a number of epileptic syndromes was then demonstrated in humans, with relatively good toler- 
ance [4] [5]. Over the next several decades, it became one of the most broadly used anticonvulsants in children worldwide. Clobazam has only recently been introduced for use in the United States with FDA approval as an adjunctive treatment of Lennox-Gastaut syndrome. In the US, the medication has been recently reviewed favorably, with a relative minimum of medication-related side effects [6]. Benzodiazepines in general have been associated with rarely causing hypothermia, especially in the elderly, but this side effect has not been reported with clobazam [7]-[9]. We report two cases of profound hypothermia associated with use of this medication for the treatment of epilepsy in therapeutic doses.

\section{Case Reports}

\subsection{Patient One}

This patient is a 3-year-old with a history of diffuse cerebral dysgenesis and resultant epilepsy. Microcephaly was recognized in the newborn period, and seizures developed, initially as infantile spasm which persisted with additional complex partial semiology. The patient had been treated with a number of medications including adrenocorticotropic hormone, valproic acid, felbamate, and newer agents such as levetiracetam and lacosamide. Current medications were felbamate, lacosamide and diazepam. Most medications failed to reduce seizure frequency significantly, and seizure clusters continued. Subsequent to introduction of clobazam in the United States the medication was introduced in a dose initially of $5 \mathrm{mg}$ daily, $0.31 \mathrm{mg} / \mathrm{kg} / \mathrm{day}$, with titration to a total dosage of $0.6 \mathrm{mg} / \mathrm{kg} /$ day. No medications changes had occurred in the months prior to clobazam introduction. Initially the parents reported significant decrease in seizure frequency, however there was associated mild sedation beyond his normal encephalopathy. Because of continued central nervous system depression he was seen in an emergency room, and in association with worsened encephalopathy, hypothermia (93F) was identified and he was admitted for evaluation and empiric treatment for possible sepsis. His inpatient evaluation, including blood, urine and spinal fluid analysis and culture, proved negative/normal for infection or other metabolic derangement. Medication levels were not checked at this admission. Temperature appeared to stabilize, and the patient was discharged home, but returned for a subsequent episode of recurrent hypothermia after 6 days. Clobazam was discontinued after this episode, on the premise it was the last medication added before onset of hypothermia, with a return to a normal temperature baseline over approximately 24 hours. Normothermia has since been maintained, and his worsened encephalopathy cleared. Reinstitution of very low-dose clobazam (2.5 mg, 0.15 $\mathrm{mg} / \mathrm{kg} /$ day) has resulted in improved seizures, and temperatures have been normal over the year since initial exposure.

\subsection{Patient Two}

The patient is a 4-year-old with a history of bacterial meningitis during the newborn period, resulting in broad areas of encephalomalacia. Her outcome was complicated by developmental delay, hemiparesis and seizures, which initially presented as infantile spasm, and were followed by convulsions, staring spells, and myoclonic jerks. The patient was treated with a wide array of medication, including valproic acid, levetiracetam, and lamotrogine. Additional medications were offered due to parental reluctance to explore resective treatments for epilepsy, and clobazam was initiated. The patient was dosed initially at $2.5 \mathrm{mg}$ twice a day $(0.25 \mathrm{mg} / \mathrm{kg} /$ day $)$, with titration to $5 \mathrm{mg}$ bid, which caused undue sedation. She was then returned to a dose of $2.5 \mathrm{mg}$ twice daily. A reduction in seizure frequency was noted, and mild sedation persisted although she was able to attend school. At ten days of therapy, she was noted to be sedate, and rectal temperature at home was $93 \mathrm{~F}$. It fell to $91 \mathrm{~F}$ by admission via emergency medical services. She was admitted to hospital, and underwent a limited sepsis evaluation with blood cultures and empiric antibiotics were administered. However, because of the close temporal association with the previous case, clobazam was considered as a possible etiology, and was discontinued. Warming ensued, and hypothermia resolved within 36 hours, and the patient's mild encephalopathy improved. She remains off clobazam at her previous baseline, with well-maintained normothermia for 12 months since initial presentation.

\section{Discussion}

Clobazam has been widely used for a number of epilepsy syndromes over the last 40 years. It is distinct from other benzodiazepines in that its molecular structure is characterized by a 1-5 orientation of nitrogen atoms 
around its heterocyclic ring, rather than a 1-4 [10]. Most analyses of safety in medication trials generally demonstrate excellent tolerance, without serious medication related difficulties [6]. Our cases however indicate hypothermia as a possible side effect in some individuals. We believe this is the first identification of a serious medical complication related to this efficacious anticonvulsant in dosing ranges previously thought to be safe. Both children had significant cerebral dysgenesis or injury, and experienced profound hypothermia in association with exposure to clobazam. Each child had been relatively medically well, without episodes of hypothermia or worsened encephalopathy with previous medication exposure or illness. Each child was admitted to hospital, and subjected to an extensive evaluation for infectious complications, on the premise that they were suffering from sepsis. This is included in one case a spinal tap and dosing with empiric antibiotics. Drug levels of medications were not obtained, possibly reflecting the lack of widespread understanding that anticonvulsant medications can be causative of temperature dysregulation.

Central control of temperature regulation is thought to result from a complex interaction between a number of hypothalamic nuclei, and both thermogenesis and cooling mechanisms are controlled therein [11]. There are therefore many potential sites for disruption of normal thermoregulation, including central inputs receiving temperature measures from the periphery or centrally; impairments in heat conserving measures such as vasoconstriction or piloerection; and impairments in heat production mechanisms such as activation of metabolism in brown fat reservoirs or shivering [11]. Tonic inhibitory GABAergic innervation to the preoptic nucleus is thought to maintain the precise control of body temperature, and inhibition of such neurons is associated with an increase in body temperature [12]. It is possible that increased activation of such neurons could result in impairments in maintaining body temperature homeostasis, especially in at risk populations, such as the elderly, or children, especially if multiple pharmacologic agents are being utilized. Clobazam, like other benzodiazepines, acts at the GABAa receptor, exerting anticonvulsant effects through allosteric modulation of the inhibitory effects of GABA [13]. In this circumstance, accentuation of GABAergic neurotransmission may interrupt normal mechanisms that maintain body temperature, resulting in increased heat loss, and hypothermia.

Other benzodiazepines have in fact been demonstrated to cause hypothermia in humans and experimental animals, although most clinical reports of serious hypothermia appear to occur in the context of intentional or inadvertent over dosage [7] [14] [15]. However, we were unable to identify any reports in the literature to date regarding clobazam and hypothermia. The hypothermic effect of benzodiazepines is well recognized and in fact has been identified as a confounding effect in studies of diazepam-induced neuroprotection after global ischemia [16]. Animal and human studies confirm a tendency for core body cooling with benzodiazepines, although the mechanism of hypothermia induction is unclear. Impairment in peripheral heat conservation responses such as piloerection and vasoconstriction has been theorized [7] [17]. Likewise, a direct effect on heat maintaining centers in the central nervous system has also been proposed [18]. Certainly children are in general at higher risk of developing hypothermia, because of a greater surface area to volume ratio when compared with adults. Additionally, both children in our report had significant cerebral dysgenesis and injury, perhaps putting them at higher risk for centrally mediated dysautonomia and temperature dysregulation. Neither one had experienced hypothermia in the past however. It is unclear how temperature depression was related to serum levels, as they were not checked in either circumstance. It is probable however, that in each case drug level was in part responsible for the temperature dysregulation, as in each circumstance a worsened encephalopathy was associated, which cleared after medication discontinuation. No medical reasons were identified that might have put them at risk for impaired clobazam metabolism. Further complicating our management, one of our patients was concurrently taking valproic acid, an anticonvulsant reported to cause hypothermia, as have other anticonvulsants [19]. This may have further impaired thermoregulation in this individual. Lastly, there are probably confounding factors that create age related or individual specific aspects that may influence the temperature dysregulation. Many reports of benzodiazepine induced hypothermia are from the extremes of age, in the young and very old. Additionally, there are conflicting reports of types of temperature dysregulation, with some indicating a heat stabilizing/retaining effect of benzodiazepines rather than heat loss [20].

One of our patients was returned to dosing with clobazam and appeared to tolerate this well at a lower dose of $0.15 \mathrm{mg} / \mathrm{kg}$, with no evidence of central nervous system depression or hypothermia. Seizure control at this lower dosage was not as good with higher dosages however. Our findings indicate that additional caution should be used in children with intractable epilepsy while using clobazam. Joint administration of other anticonvulsants that might produce hypothermia should be a consideration as well. In these circumstances, lower dosages per kilogram may be reasonable, with slower titration as tolerated. Monitoring for central nervous system depression, 
and temperature dysregulation is indicated, and parental counseling likewise paramount.

\section{Conclusion}

These cases indicate that episodes of hypothermia may be associated with clobazam in children. Consideration of other possibilities including infectious etiologies should always be considered, especially in children with neurologic compromise, as they are prone to infectious complications. However, medication effects should be considered in the differential diagnosis of unexplained hypothermia. Although anecdotal, there may be a greater propensity for such side effects in children with central nervous system injury whether congenital or acquired, and greater caution might be exercised in these circumstances as well. Lastly, the mechanism of hypothermia remains unclear, given reports of both abnormal temperature elevation and depression with benzodiazepines. Further study is warranted, and correlation with drug levels in future patients would help clarify if this is an idiosyncratic response or due to elevated serum concentrations.

\section{Author Contributions}

Marc P. DiFazio—draft preparation, outpatient care for both patients reported.

Bennett Lavenstein — draft revisions, attending inpatient service caring for both patients.

Scott Lavenstein—draft revisions, neurology fellow on inpatient service caring for both patients.

\section{Conflict of Interest}

The authors have no conflicts of interest.

\section{Ethical Approval}

There is no need for IRB approval at Children’s National Medical for case reports.

\section{References}

[1] Birkmayer, W. (1979) Clinical Experiences with Clobazam. British Journal of Clinical Pharmacology, 7, 121S-122S. http://dx.doi.org/10.1111/j.1365-2125.1979.tb04678.x

[2] Fielding, S. and Hoffmann, I. (1979) Pharmacology of Anti-Anxiety Drugs with Special Reference to Clobazam. British Journal of Clinical Pharmacology, 7, 7S-15S. http://dx.doi.org/10.1111/j.1365-2125.1979.tb05920.x

[3] Borland, R.G. and Nicholson, A.N. (1975) Immediate Effects on Human Performance of a 1,5-Genzodiazepine (Clobazam) Compared with the 1,4-Benzodiazepines, Chlordiazepoxide Hydrochloride and Diazepam. British Journal of Clinical Pharmacology, 2, 215-221. http://dx.doi.org/10.1111/j.1365-2125.1975.tb01578.x

[4] Barzaghi, F., Fournex, R. and Mantegazza, P. (1973) Pharmacological and Toxicological Properties of Clobazam (1-Phenyl-5-methyl-8-chloro-1,2,4,5-tetrahydro-2,4-diketo-3H-1,5-benzodiazepine), a New Psychotherapeutic Agent. Arzneimittelforschung, 23, 683-686.

[5] Gastaut, H. and Low, M.D. (1979) Antiepileptic Properties of Clobazam, a 1-5 Benzodiazepine, in Man. Epilepsia, 20, 437-446. http://dx.doi.org/10.1111/j.1528-1157.1979.tb04825.x

[6] Wheless, J.W. and Phelps, S.J. (2013) Clobazam: A Newly Approved but Well-Established Drug for the Treatment of Intractable Epilepsy Syndromes. Journal of Child Neurology, 28, 219-229. http://dx.doi.org/10.1111/j.1528-1157.1979.tb04825.x

[7] Clark, S.M. and Lipton, J.M. (1981) Effects of Diazepam on Body Temperature of the Aged Squirrel Monkey. Brain Research Bulletin, 7, 5-9. http://dx.doi.org/10.1016/0361-9230(81)90091-5

[8] Whitelaw, A.G., Cummings, A.J. and McFadyen, I.R. (1981) Effect of Maternal Lorazepam on the Neonate. British Medical Journal (Clinical Research ed.), 282, 1106-1108. http://dx.doi.org/10.1136/bmj.282.6270.1106

[9] Echizenya, M., Mishima, K., Satoh, K., et al. (2004) Enhanced Heat Loss and Age-Related Hypersensitivity to Diazepam. Journal of Clinical Psychopharmacology, 24, 639-646. http://dx.doi.org/10.1097/01.jcp.0000144890.45234.e9

[10] Muthu, S., Prasath, M. and Arun Balaji, R. (2011) Experimental and Theoretical Investigations of Spectroscopic Properties of Clobazam. Recent Research in Science and Technology, 3, 127-135.

[11] Clapham, J.C. (2012) Central Control of Thermogenesis. Neuropharmacology, 63, 111-123. http://dx.doi.org/10.1016/j.neuropharm.2011.10.014

[12] Dimitrov, E.L., Kim, Y.Y. and Usdin, T.B. (2011) Regulation of Hypothalamic Signaling by Tuberoinfundibular Pep- 
tide of 39 Residues Is Critical for the Response to Cold: A Novel Peptidergic Mechanism of Thermoregulation. Journal of Neuroscience, 31, 18166-18179. http://dx.doi.org/10.1523/JNEUROSCI.2619-11.2011

[13] Sankar, R. (2012) GABA(A) Receptor Physiology and Its Relationship to the Mechanism of Action of the 1,5-Benzodiazepine Clobazam. CNS Drugs, 26, 229-244. http://dx.doi.org/10.2165/11599020-000000000-00000

[14] Irvine, R.E. (1966) Hypothermia Due to Diazepam. British Medical Journal, 2, 1007. http://dx.doi.org/10.1136/bmj.2.5520.1007

[15] Hostler, D., Northington, W.E. and Callaway, C.W. (2009) High-Dose Diazepam Facilitates Core Cooling during Cold Saline Infusion in Healthy Volunteers. Applied Physiology, Nutrition, and Metabolism, 34, 582-586. http://dx.doi.org/10.1139/H09-011

[16] Dowden, J., Reid, C., Dooley, P. and Corbett, D. (1999) Diazepam-Induced Neuroprotection: Dissociating the Effects of Hypothermia Following Global Ischemia. Brain Research, 829, 1-6. http://dx.doi.org/10.1016/S0006-8993(99)01229-9

[17] Kurz, A., Sessler, D.I., Annadata, R., et al. (1995) Midazolam Minimally Impairs Thermoregulatory Control. Anesthesia \& Analgesia, 81, 393-398.

[18] Vidal, C., Suaudeau, C. and Jacob, J. (1983) Hyper- and Hypothermia Induced by Non-Noxious Stress: Effects of Naloxone, Diazepam and Gamma-Acetylenic GABA. Anesthesia \& Analgesia, 33, 587-590.

[19] Zachariah, S.B., Zachariah, A., Ananda, R. and Stewart, J.T. (2000) Hypothermia and Thermoregulatory Derangements Induced by Valproic Acid. Neurology, 55, 150-151. http://dx.doi.org/10.1212/WNL.55.1.150

[20] Hess, P.E., Snowman, C.E. and Wang, J. (2005) Hypothermia after Cesarean Delivery and Its Reversal with Lorazepam. International Journal of Obstetric Anesthesia, 14, 279-283. http://dx.doi.org/10.1016/j.ijoa.2005.02.004 